\title{
The usefulness of SPECT-CT with radioisotope-labeled leukocytes in diagnosing lead-dependent infective endocarditis
}

\author{
Barbara A. Małecka ${ }^{1,2, A, B, B, D, F}$, Andrzej Ząbek ${ }^{1, B, C, E, F}$, Maciej Dębski ${ }^{1, B, E, F}$, Wojciech Szot ${ }^{3,4, B, B, F}$, Katarzyna Holcman ${ }^{5, B, E, F}$, \\ Krzysztof Boczar ${ }^{1, B, E, F}$, Mateusz Ulman ${ }^{1, B, E, F}$, Jacek Lelakowski ${ }^{1,2, B, E, F}$, Magdalena Kostkiewicz ${ }^{2,5, B, E, F}$ \\ ${ }^{1}$ Department of Electrocardiology, John Paul II Hospital, Kraków, Poland \\ ${ }^{2}$ Institute of Cardiology, Jagiellonian University Medical College, Kraków, Poland \\ ${ }^{3}$ Department of Hygiene and Dietetics, Jagiellonian University Medical College, Kraków, Poland \\ ${ }^{4}$ Nuclear Medicine Department, John Paul II Hospital, Kraków, Poland \\ ${ }^{5}$ Department of Cardiac and Vascular Diseases, John Paul II Hospital, Kraków, Poland \\ A - research concept and design; $B$ - collection and/or assembly of data; $C$ - data analysis and interpretation; \\ $D$ - writing the article; $E$ - critical revision of the article; $F$ - final approval of the article
}

\section{Address for correspondence \\ Andrzej Ząbek \\ E-mail:andrzej_j_z@poczta.onet.pl}

\section{Funding sources}

None declared

Conflict of interest

None declared

Received on February 22, 2018

Reviewed on March 28, 2018

Accepted on June 15, 2018

Published online on October 31, 2018

Cite as

Małecka B, Ząbek A, Dębski M, et al. The usefulness of SPECT-CT with radioisotope-labeled leukocytes in diagnosing lead-dependent infective endocarditis. Adv Clin Exp Med. 2019;28(1):113-119. doi:10.17219/acem/92315

DOI

10.17219/acem/92315

Copyright

Copyright by Author(s)

This is an article distributed under the terms of the

Creative Commons Attribution Non-Commercial License

(http://creativecommons.org/licenses/by-nc-nd/4.0/)

\begin{abstract}
Background. Lead-dependent infective endocarditis (LDIE) is a life-threatening complication of permanent transvenous cardiac pacing. According to the 2015 European Society of Cardiology (ECS) guidelines, the diagnosis of LDIE is based on the modified Duke criteria (MDC), while single-photon emission computed tomography with conventional computed tomography (SPECT-CT) with radioisotope-labeled leukocytes serves as an additional tool in difficult cases. The major challenge is to differentiate between true vegetation and a thrombus.
\end{abstract}

Objectives. The aim of the study was to evaluate the usefulness of SPECT-CT with radioisotope-labeled leukocytes in diagnosing LDIE in patients with intracardiac masses (ICMs).

Material and methods. The prospective registry included 40 consecutive patients admitted with an ICM on the lead and suspicion of LDIE. The confirmation or rejection of the LDIE diagnosis was made according to an algorithm based on the MDC. The cohort was divided into 2 groups: patients with definite and possible LDIE diagnoses based on the MDC (the LDIE-positive group), and patients with negative LDIE diagnoses according to the MDC (the LDIE-negative group). All patients underwent SPECT-CT with radioisotope-labeled leukocytes. The diagnostic ability of SPECT-CT was compared to the gold standard MDC.

Results. The LDIE-positive group with diagnosis based on the MDC consisted of 19 patients (LDIE definite - 11; LDIE possible - 8). The LDIEdiagnosis was rejected on the basis of the MDC in 21 patients. The SPECT-CT results were compared with the MDC results and showed $73.7 \%$ sensitivity, $81.0 \%$ specificity, $77.5 \%$ accuracy, $77.8 \%$ positive predictive value (PPV), $77.3 \%$ negative predictive value (NPV), likelihood ratio positive (LR+) 3.868, likelihood ratio negative ( $(R-) 0.325$, and moderate agreement $(K=0.548, p<0.001)$. After the exclusion of 5 patients treated with antibiotics at the time of the SPECT-CT, LR+ and LR-improved to 5.250 and 0 , respectively, and inter-test agreement amounted to almost perfect concordance $(K=0.773, p<0.001)$.

Conclusions. Single-photon emission computed tomography with conventional CT with radioisotopelabeled leukocytes is a useful, efficient, single-step test for diagnosing LDIE.

Key words: scintigraphy, vegetation, infective endocarditis, cardiac pacing, artificial, radionuclide imaging 


\section{Introduction}

Lead-dependent infective endocarditis (LDIE) is a lifethreatening complication of permanent transvenous cardiac pacing occurring in the right side of the heart. The term was coined to underline the fact that LDIE is a unique disease process and a distinct entity in the wide spectrum of cardiac device-related infective endocarditis (CDRIE), in which inflammation is associated with various types of implantable devices. ${ }^{1}$ According to most of the available reports, LDIE is the major risk factor for mortality after transvenous lead extraction (TLE) procedures. ${ }^{2}$ The 2009 guidelines of European Society of Cardiology (ESC) concerning the prevention, diagnosis and treatment of infective endocarditis (IE) outlined straightforward criteria (the Duke criteria) that should be met in order to diagnose IE. ${ }^{3}$ The major criteria for diagnosing IE include positive blood cultures and echocardiographic findings characteristic of IE, such as vegetation and abscess formation. To identify patients with indwelling endocardial leads, 2 additional major criteria have been introduced: local signs of infection and pulmonary embolism.

Implementing the results of additional imaging investigations of the source of infection - positron emission tomography/computed tomography (PET-CT) and singlephoton emission computed tomography with conventional computed tomography (SPECT-CT) with radioisotopelabeled leukocytes - into the ESC guidelines and giving them the importance of major criteria may significantly improve the diagnostic accuracy of the Duke criteria. ${ }^{4}$ The role of nuclear medicine, according to the guidelines, is confined to the diagnosis of prosthetic valve endocarditis. The authors of the guidelines mentioned that PET-CT and SPECT-CT have proven their role in the diagnosis of cardiac implantable electronic devices (CIEDs), but the data is not sufficient for them to be included in the diagnostic criteria of the specific topic of IE on pacemaker or defibrillator leads. ${ }^{4}$ Notably, in the chapter on diagnosing cardiac device IE, the authors recognize the utility of SPECT-CT and PET-CT scanning as additional tools in difficult cases, such as in patients with suspected LDIE, positive blood cultures and negative echocardiography (Class IIb, level of evidence C). ${ }^{4}$ Erba et al. showed that SPECT-CT allowed LDIE to be confirmed or reliably excluded device-associated infections during febrile episodes and sepsis, with $95 \%$ negative predictive value (NPV). ${ }^{5}$

The aim of the present study was to show the diagnostic value of SPECT-CT in patients with an intracardiac mass (ICM) suspected of being vegetation, in comparison to the gold standard modified Duke criteria (MDC).

\section{Material and methods}

The prospective registry included consecutive patients with ICMs on the lead admitted to a reference university center (Department of Electrocardiology, John Paul II Hospital, Kraków, Poland) from August 2014 to August 2017. The prerequisites for including a patient in the study were: detection of an ICM on echocardiography and provision of informed consent to participate in the study. Confirmation or rejection of an LDIE diagnosis was made according to the algorithm used in our center and based on the MDC, which is considered the gold standard (Fig. 1). Among the patients, there was a variety of clinical presentations and various degrees of clinical IE suspicion (Table 1). A final diagnosis of LDIE according to the MDC was established after collecting all the tests included in the major and minor criteria. All the patients also underwent SPECT-CT scanning.

The cohort was divided into 2 groups: patients with definite and possible LDIE diagnoses based on the MDC (the LDIE-positive group), and patients with negative

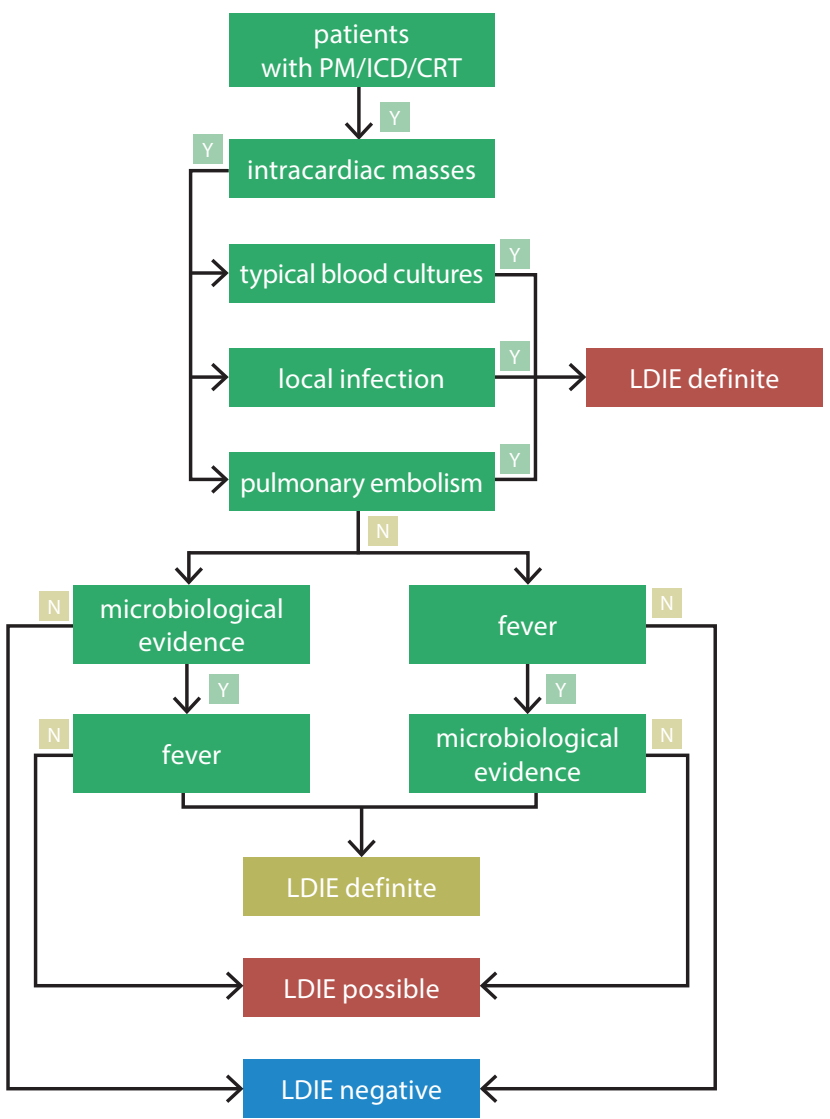

Fig. 1. The diagnostic algorithm for the diagnosis of lead-dependent infective endocarditis (LDIE) based on the modified Duke criteria (MDC). $\mathrm{Y}$-yes, $\mathrm{N}$ - no; typical blood cultures: blood cultures for microorganisms consistent with infective endocarditis (IE) from 2 or more separate cultures of blood were treated as major criterion; microbiological evidence: positive blood culture but does not meet major criterion, more than 1 positive blood culture with skin bacteria was treated as a minor criterion, 1 positive blood culture with skin bacteria was treated as sample contamination; local infection: signs of inflammation of the pocket of the cardiac device or pocket skin erosion with purulent drainage; septic pulmonary embolism: clinical, echocardiographic and laboratory features of pulmonary embolism accompanied by evidence of recurrent pulmonary infections

PM - pacemaker; ICD - implantable cardioverter-defibrillator;

CRT - cardiac resynchronization therapy. 
LDIE diagnoses according to the MDC (the LDIE-negative group). Both groups included patients with positive and negative SPECT-CT results. The diagnostic test evaluated in the present study was SPECT-CT, which was compared with the gold standard MDC.

Approval to conduct the study was obtained from the local ethics committee.

\section{SPECT-CT as a diagnostic modality}

In our center, the autologous leukocyte labeling procedure was performed in strict accordance with the Society of Nuclear Medicine Procedure Guidelines. ${ }^{6}$ Whole-body scans followed by chest SPECT-CT scans were acquired $6 \mathrm{~h}$ and $24 \mathrm{~h}$ after the injection of radioisotope-labeled white blood cells (WBCs) with the use of a Symbia T16 SPECT-CT gamma camera system (Siemens AG, Munich, Germany). The first 10 patients underwent SPECT-CT with Scintimun ${ }^{\circledR}$ (Cisbio, Codolet, France) and subsequent patients with ${ }^{99 m}$ Tc-HMPAO (GE Healthcare Ltd., Amersham, UK). The transmission data were reconstructed using filtered back projection to produce cross-sectional images. The resolution of the computed tomography (CT) scan was $2.5 \mathrm{~mm}$, and localization images were produced with a 4.5-mm pixel size, similar to nuclear medicine emission images. The CT scans were reconstructed onto a $256 \times 256$ matrix. The SPECT component of the same field of view was acquired using a $128 \times 128$ matrix, $360^{\circ}$ rotation, $6^{\circ}$ angle step, and acquisition time of $25 \mathrm{~s}$ per frame. Both attenuation-corrected CT and noncorrected SPECT images were evaluated in the coronal, transaxial and sagittal plane modes. All the studies were evaluated by 2 experienced nuclear medicine specialists. Scintigraphy was considered positive for CDRIE when an area of labeled WBCs uptake superior to the background activity was identified in the involved area and when the signal increased over time (Fig. 2). ${ }^{7}$

\section{Statistical analysis}

The statistical analysis was performed using the STATISTICA v. 12.5 data analysis software system (StatSoft Inc., Tulsa, USA). For quantitative variables, minimum, maximum, mean, and standard deviation (SD) values were provided. The results of the tests were expressed as a 2-way contingency table. The assessment of the tests included the following parameters: sensitivity, specificity, positive predictive value (PPV), negative predictive value (NPV), accuracy, likelihood ratio for a positive test result (LR+), and likelihood ratio for a negative test result (LR-).

The interpretation of the likelihood ratios (LRs) was performed according to Attia. ${ }^{8}$ A test has real diagnostic utility if the LR is $\geq 10$ or $\leq 0.1$. Values between 5 and 10 and between 0.1 and 0.2 show that a test is moderately useful. An LR between 0.5 and 2 indicates that the test has no diagnostic value. ${ }^{9}$ Inter-observer variability was calculated using multi-rater Cohen's kappa (к) statistics with
Table 1. Indications for echocardiography in consecutive patients with ICM: clinical presentation, diagnosis based on the Duke criteria and the results of SPECT-CT studies

\begin{tabular}{|c|c|c|c|c|}
\hline $\begin{array}{c}\text { Patient } \\
\text { No. }\end{array}$ & $\begin{array}{c}\text { Clinical } \\
\text { presentation }\end{array}$ & $\begin{array}{c}\text { Diagnosis } \\
\text { of LDIE based } \\
\text { on Duke criteria }\end{array}$ & $\begin{array}{l}\text { SPECT-CT } \\
\text { heart }\end{array}$ & $\begin{array}{l}\text { SPECT-CT } \\
\text { pocket }\end{array}$ \\
\hline 1. & A & $\mathrm{N}$ & $\mathrm{N}$ & $\mathrm{N}$ \\
\hline 2. & B & D & $\mathrm{N}$ & P \\
\hline 3. & C & $D$ & $\mathrm{~N}$ & $\mathrm{~N}$ \\
\hline 4. & DT & $\mathrm{N}$ & $\mathrm{N}$ & $N$ \\
\hline 5. & DT & $\mathrm{N}$ & $N$ & $\mathrm{~N}$ \\
\hline 6. & A & $\mathrm{N}$ & $N$ & $N$ \\
\hline 7. & $A$ & Ps & $P$ & $\mathrm{~N}$ \\
\hline 8. & $A$ & Ps & $P$ & $\mathrm{~N}$ \\
\hline 9. & $B$ & $D$ & $\mathrm{~N}$ & $\mathrm{~N}$ \\
\hline 10. & $A$ & $D$ & $P$ & $\mathrm{~N}$ \\
\hline 11. & $C$ & $D$ & $P$ & $\mathrm{~N}$ \\
\hline 12. & A & Ps & $P$ & $\mathrm{~N}$ \\
\hline 13. & $B$ & $\mathrm{D}$ & $P$ & $\mathrm{~N}$ \\
\hline 14. & A & $N$ & P & P \\
\hline 15. & $A$ & $N$ & $P$ & $\mathrm{~N}$ \\
\hline 16. & $A$ & $N$ & $N$ & $N$ \\
\hline 17. & $C$ & $D$ & $P$ & $\mathrm{~N}$ \\
\hline 18. & DT & $N$ & $\mathrm{~N}$ & $\mathrm{~N}$ \\
\hline 19. & $A$ & $N$ & $\mathrm{~N}$ & $\mathrm{~N}$ \\
\hline 20. & $C$ & D & $P$ & $\mathrm{~N}$ \\
\hline 21. & $E$ & Ps & $N$ & $\mathrm{~N}$ \\
\hline 22. & $A$ & $\mathrm{~N}$ & $P$ & $\mathrm{~N}$ \\
\hline 23. & A & $\mathrm{N}$ & $\mathrm{N}$ & $\mathrm{N}$ \\
\hline 24. & $A$ & Ps & $P$ & $\mathrm{~N}$ \\
\hline 25. & $A$ & Ps & $P$ & $\mathrm{~N}$ \\
\hline 26. & B & $D$ & $N$ & $\mathrm{~N}$ \\
\hline 27. & DT & $\mathrm{N}$ & $P$ & $\mathrm{~N}$ \\
\hline 28. & A & $\mathrm{N}$ & $N$ & $\mathrm{~N}$ \\
\hline 29. & DT & $\mathrm{N}$ & $\mathrm{N}$ & $\mathrm{N}$ \\
\hline 30. & A & $\mathrm{N}$ & $\mathrm{N}$ & $\mathrm{N}$ \\
\hline 31. & $E$ & Ps & P & $\mathrm{N}$ \\
\hline 32. & $E$ & $\mathrm{~N}$ & $\mathrm{~N}$ & $N$ \\
\hline 33. & $C$ & $D$ & P & $\mathrm{N}$ \\
\hline 34. & $A$ & $N$ & $N$ & $\mathrm{~N}$ \\
\hline 35. & $A$ & $\mathrm{~N}$ & $\mathrm{~N}$ & $\mathrm{~N}$ \\
\hline 36. & $B$ & $D$ & $P$ & $P$ \\
\hline 37. & DT & $\mathrm{N}$ & $\mathrm{N}$ & $\mathrm{N}$ \\
\hline 38. & $E$ & Ps & $P$ & P \\
\hline 39. & $A$ & $\mathrm{~N}$ & $\mathrm{~N}$ & $\mathrm{~N}$ \\
\hline 40. & $A$ & $\mathrm{~N}$ & $\mathrm{~N}$ & $\mathrm{~N}$ \\
\hline
\end{tabular}

A - diagnostic work-up of dyspnoea and/or heart failure; B - diagnostic work-up prior to transvenous lead extraction due to pocket infection; C - diagnostic work-up of sepsis; DT - diagnostic work-up prior to transvenous lead extraction due to lead dysfunction/system change/system upgrade; E - diagnostic work-up of fever; $\mathrm{N}$ - negative; P - positive; PS - possible; D - definite; ICM - intracardiac mass; LDIE - lead-dependent infective endocarditis; SPECT-CT - single-photon emission computed tomography with conventional computed tomography. 

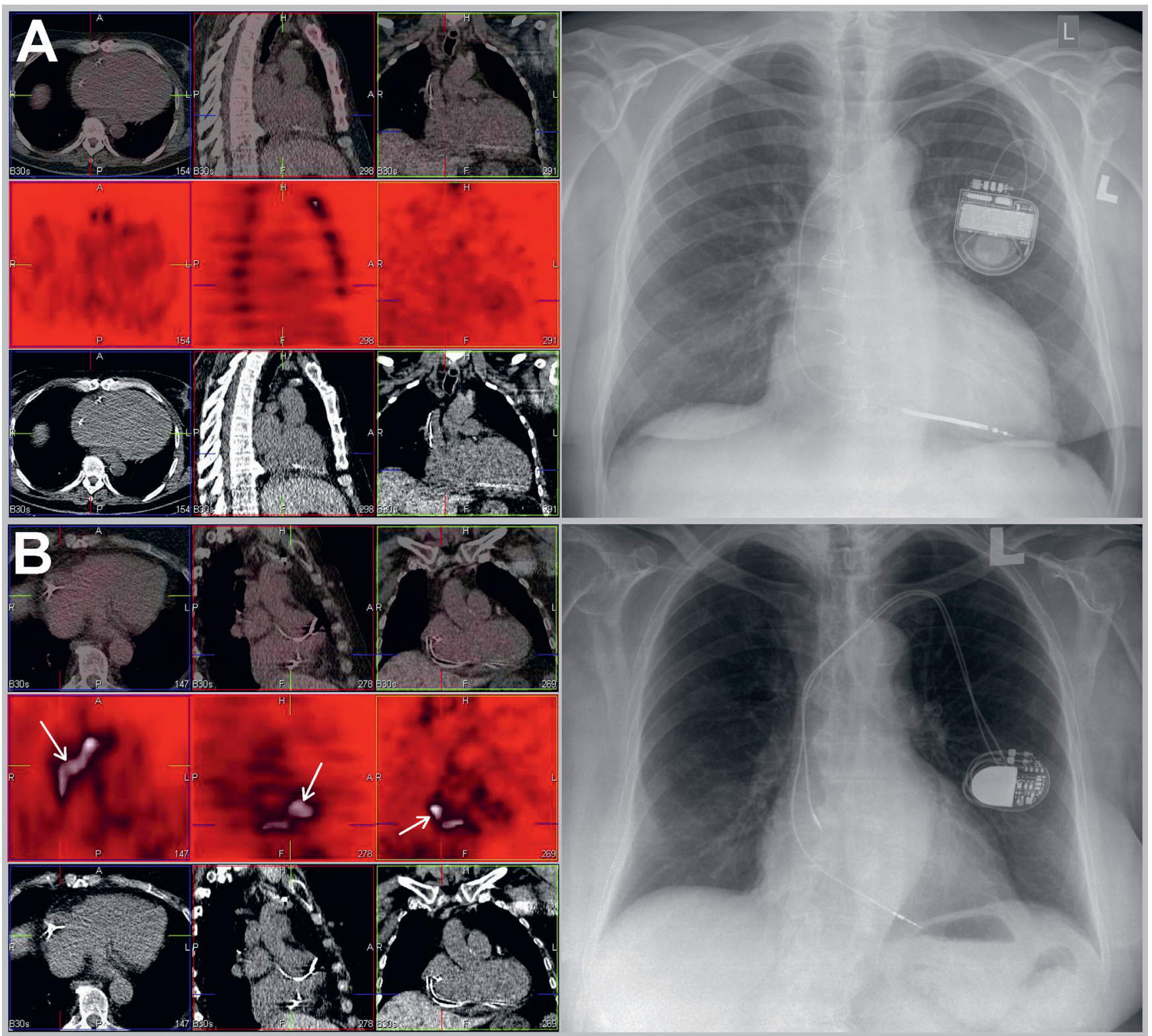

Fig. 2. Single-photon emission computed tomography with conventional computed tomography (SPECT-CT) and chest X-ray in patients with negative and positive SPECT-CT results. A - Single-chamber implantable cardioverter-defibrillators (ICD) system. The SPECT-CT study with the use of ${ }^{99 m}$ Tc-HMPAOlabeled leukocytes on the left side of the picture and chest X-ray picture on the right side. The negative result of the SPECT-CT study is presented in the upper and middle panels of the SPECT-CT figure. B - Dual-chamber pacemaker (DDD) pacing system. SPECT-CT study with the use of 99mTc-HMPAOlabeled leukocytes on the left side of the picture and chest X-ray picture on the right side. Positive result suggesting infective endocarditis (IE) is presented in the upper and middle panels of the SPECT-CT figure. Focal uptake observed in the right atrium and partially in the right ventricle near the lead (arrows)

a $95 \%$ confidence interval (CI). The definitions presented by Landis and Koch were used to evaluate the strength of the rater agreement and were categorized as slight (0-0.20); fair (0.21-0.40); moderate (0.41-0.60); substantial (0.61-0.80); and almost perfect $(0.81-1.00) .{ }^{10} \mathrm{~A} 2$-tailed p-value $<0.05$ was considered significant.

\section{Results}

The study population consisted of 40 patients ( $7 \mathrm{fe}$ males and 33 males), mean age $62.0 \pm 16.5$ years (range: 23.8-89.0), with different types of CIEDs and ICMs detected by transthoracic or transesophageal echocardiography (TTE/TEE). The patients were implanted with the following types of CIED: 19 had pacemakers, 12 had implantable cardioverter-defibrillators (ICD), 7 had undergone cardiac resynchronization therapy (CRT), and 2 had pacemakers and ICDs on both sides of the chest. Lead dwell time was $102.1 \pm 85.5$ months (range: $0.6-434.1$ ).

In the LDIE-positive group, there were 19 patients (3 female) with an average age of $72.6 \pm 8.9$ years. Definite LDIE was diagnosed in 11 patients on the basis of at least 2 major criteria fulfilled: along with ICMs there was septic pulmonary embolism in 6 patients and local infection in 5 patients. Two patients with definite LDIE had positive blood cultures 
for Staphylococcus aureus, thus meeting a major Duke criterion. Possible LDIE was diagnosed in 8 patients in the presence of 1 major and 1 minor criterion (Table 1). The major Duke criterion in each of these patients was an ICM; the minor criteria were fever in 6 patients and positive blood cultures that did not meet the major Duke criterion in 2 patients.

In the LDIE-negative group, there were 21 patients ( $4 \mathrm{fe}$ male) with an average age of $57.9 \pm 21.8$ years. When compared with the MDC results, the results obtained by SPECT-CT were true positive (TP) in 14 patients, false negative (FN) in 5 , false positive (FP) in 4 , and true negative (TN) in 17 patients. The SPECT-CT results were FN in 5 patients in whom antibiotic treatment had been administered before the examination. Based on the number of patients with TP, FN, FP, and TN results, the diagnostic value of SPECT-CT in relation to MDC was calculated (Table 2). Diagnostic test using SPECT-CT showed high sensitivity, specificity and accuracy $(73.7 \%, 81.0 \%$ and $77.5 \%$, respectively) and high PPV and NPV (PPV 77.8\% and NPV 77.3\%). The scintigraphic test can be useful to diagnose or rule out LDIE (LR+ = 3.868, LR $-=0.325$ ). Agreement between the 2 tests was moderate but statistically significant $(\mathrm{K}=0.548, \mathrm{p}<0.001)$, according to Landis and Koch. ${ }^{10}$

After the exclusion of the 5 patients undergoing antibiotic treatment at the time of the examination, the SPECT-CT test sensitivity, accuracy and NPV significantly increased

Table 2. The diagnostic value of SPECT-CT in relation to MDC

\begin{tabular}{|c|c|c|}
\hline \multicolumn{2}{|c|}{ Parameter } & \multirow{2}{*}{$\begin{array}{c}\text { Test based on SPECT-CT } \\
73.7 \%\end{array}$} \\
\hline & result & \\
\hline & $95 \% \mathrm{Cl}$ & $55.1-86.1 \%$ \\
\hline \multirow{2}{*}{ Specificity } & result & $81.0 \%$ \\
\hline & $95 \% \mathrm{Cl}$ & $64.2-92.2 \%$ \\
\hline \multirow{2}{*}{ PPV } & result & $77.8 \%$ \\
\hline & $95 \% \mathrm{Cl}$ & $58.2-90.9 \%$ \\
\hline \multirow{2}{*}{ NPV } & result & $77.3 \%$ \\
\hline & $95 \% \mathrm{Cl}$ & $61.3-88.0 \%$ \\
\hline \multirow{2}{*}{ ACC } & result & $77.5 \%$ \\
\hline & $95 \% \mathrm{Cl}$ & $59.9-89.3 \%$ \\
\hline \multirow{2}{*}{$\mathrm{LR}+$} & result & 3.868 \\
\hline & $95 \% \mathrm{Cl}$ & $1.539-11.065$ \\
\hline \multirow{2}{*}{ LR- } & result & 0.325 \\
\hline & $95 \% \mathrm{Cl}$ & $0.150-0.699$ \\
\hline \multirow{5}{*}{ K } & k & 0.548 \\
\hline & SE & 0.133 \\
\hline & $95 \% \mathrm{Cl}$ & $0.193-0.785$ \\
\hline & Z & 3.447 \\
\hline & $p$-value & $<0.001$ \\
\hline
\end{tabular}

$\mathrm{Cl}$ - confidence interval; PPV - positive predictive value; NPV - negative predictive value; ACC - accuracy; LR+ - likelihood ratio for a positive test result; LR - - likelihood ratio for a negative test result; $M D C$ - modified Duke criteria; SPECT-CT - single-photon emission computed tomography with conventional computed tomography; $\mathrm{k}$ - multi-rater Cohen's kappa; SE - standard error; Z - z-score.
Table 3. The diagnostic value of SPECT-CT for MDC after exclusion of 5 patients with false negative (FN) results due to ongoing antibiotic treatment at the time of the SPECT-CT

\begin{tabular}{|c|c|c|}
\hline \multicolumn{2}{|c|}{ Parameter } & Test based on SPECT-CT \\
\hline \multirow{2}{*}{ Sensitivity } & result & $100.0 \%$ \\
\hline & $95 \% \mathrm{Cl}$ & $79.0-100.0 \%$ \\
\hline \multirow{2}{*}{ Specificity } & result & $81.0 \%$ \\
\hline & $95 \% \mathrm{Cl}$ & $67.0-81.0 \%$ \\
\hline \multirow{2}{*}{ PPV } & result & $77.8 \%$ \\
\hline & $95 \% \mathrm{Cl}$ & $61.5-77.8 \%$ \\
\hline \multirow{2}{*}{ NPV } & result & $100.0 \%$ \\
\hline & $95 \% \mathrm{Cl}$ & $82.7-100.0 \%$ \\
\hline \multirow{2}{*}{ ACC } & result & $88.6 \%$ \\
\hline & $95 \% \mathrm{Cl}$ & $71.8-88.6 \%$ \\
\hline \multirow{2}{*}{ LR+ } & result & 5.250 \\
\hline & $95 \% \mathrm{Cl}$ & $2.391-5.250$ \\
\hline \multirow{2}{*}{ LR- } & result & 0.000 \\
\hline & $95 \% \mathrm{Cl}$ & $0.000-0.313$ \\
\hline \multirow{5}{*}{ K } & K & 0.773 \\
\hline & SE & 0.107 \\
\hline & $95 \% \mathrm{Cl}$ & $0.439-0.773$ \\
\hline & Z & 4.598 \\
\hline & $p$-value & $<0.001$ \\
\hline
\end{tabular}

$\mathrm{Cl}$ - confidence interval; PPV - positive predictive value; NPV - negative predictive value; $A C C$ - accuracy; LR+ - likelihood ratio for a positive test result; LR - - likelihood ratio for a negative test result; MDC - modified Duke criteria; SPECT-CT - single-photon emission computed tomography with conventional computed tomography; $\mathrm{k}$ - multi-rater Cohen's kappa; SE - standard error; Z - Z-score.

to $100 \%, 88.6 \%$ and $100 \%$, respectively (Table 3 ). Furthermore, LR+ amounted to 5.250 and LR- reached 0; agreement between the 2 tests improved to almost perfect concordance $(\kappa=0.773, \mathrm{p}<0.001) .{ }^{10}$

\section{Discussion}

The detection of an ICM in a patient with indwelling endocardial leads requires a complete diagnostic workup to identify or exclude infection of the endocardium. A lack of other symptoms of inflammation leaves clinicians in uncertainty, because LDIE can have an oligosymptomatic course and non-characteristic symptoms. ${ }^{11}$ On the other hand, the presence of an implanted lead can promote thrombus formation. ${ }^{12,13}$ Therefore, accurate clinical judgment is necessary to detect pulmonary embolism caused by uninfected lead-related thrombi, which should not be considered the major Duke criterion of LDIE.

The available literature supplies vast evidence of the difficulties in real-world clinical practice regarding diagnosing IE in patients with CIEDs using the MDC.

Polewczyk et al., conducting research in a single patient cohort, did not differentiate definite from possible LDIE 
in one study, but introduced this division in a subsequent report. ${ }^{14,15}$ In the first study, the authors used their own modification of the Duke criteria to allow for a diagnosis of definite LDIE when 1 major and 2 minor criteria were satisfied. In the subsequent study, they used the criteria proposed by the ESC; however, the total number of diagnosed IE cases did not change. Furthermore, the authors did not provide information on the method used to diagnose LDIE in $1 / 3$ of the patients without vegetation in the assessed group of 500 patients; however, they drew significant conclusions about the different mechanisms of LDIE development in these patients. ${ }^{16}$

We presented the diagnostic scheme adopted in our institution to confirm possible and definite LDIE in patients with CIEDs using the MDC. The detection of LDIE based on this interpretation of the Duke criteria, the gold standard of IE diagnosis, allowed us to evaluate the utility of using an accessory imaging modality such as SPECT-CT in the diagnostic workup of LDIE. A high correlation was observed in the results obtained using the 2 tests in the diagnosis of LDIE. In 5 patients with definite LDIE diagnoses who had received antibiotic treatment before admission to our institute, the SPECT-CT result was FN. Similar observations have been reported in the literature. The initiation of antimicrobial treatment before the termination of the diagnostic workup was the probable cause of the FN results of SPECT-CT and PET. ${ }^{17,18}$

The primary difficulty we encountered when attempting to compare our observations with other reports on patients referred for TLE procedures is the lack of consistency in the application of the Duke criteria in real-world clinical practice. In a French study, the authors acknowledged a positive lead culture and permanently positive bacterial culture with pathogens not consistent with IE as the major microbiological Duke criterion, which is discordant with ESC guidelines. ${ }^{17}$

We demonstrated the high sensitivity, specificity and accuracy of SPECT-CT scans in LDIE diagnosis (73.7\%, 81.0\% and $77.5 \%$, respectively), with high positive and negative predictive values (77.8\% and $77.3 \%$, respectively). Our outcomes differed from the results obtained by positron emission tomography (PET) testing in a study by Cautela et al., who reported significantly lower sensitivity and specificity in LDIE detection (30.8\% and $62.5 \%$, respectively). ${ }^{17}$ The discrepancy in the reported results of diagnostic tests might have been due to the adoption of different criteria to diagnose LDIE. A recent meta-analysis of 6 studies using fluorine-18-fluorodeoxyglucose positron emission tomography/computed tomography $\left({ }^{18} \mathrm{~F}-\mathrm{FDG}\right.$ PET-CT) reported a pooled sensitivity of $65 \%$ and specificity of $88 \%$ for lead-dependent CIED infections, which concurs with our results. ${ }^{19}$

In our cohort, the agreement between SPECT-CT and MDC results according to Landis and Koch was moderate, but statistically significant. ${ }^{10}$ Importantly, when analyzing patients who had not been treated with antibiotics before SPECT-CT, the agreement between the 2 tests was almost perfect. The scintigraphic test can be useful to diagnose or rule out LDIE $(\mathrm{LR}+=3.868, \mathrm{LR}-=0.325)$. The implementation of SPECT-CT in the ESC guidelines has enabled precise diagnoses in difficult cases, such as in patients with isolated ICMs, and helped to avoid the risk of serious complications associated with TLE procedures.

Conducting a single imaging test is an advantage of using SPECT-CT, whereas diagnostic evaluation using the MDC requires multiple blood cultures and TTE/TEE assessments.

One limitation of the present study is the relatively small sample size; however, the results did not differ significantly from other similar reports. ${ }^{19}$ The radiotracer used in SPECT-CT was not uniform in the whole cohort: the first 10 patients underwent assessment with Scintimun ${ }^{\circledR}$ and subsequent patients with ${ }^{99 \mathrm{~m}} \mathrm{Tc}-\mathrm{HMPAO}$. These 2 methods have not been compared in the diagnosis of IE, but in the authors' opinion, the use of 2 radiotracers did not add much bias, because only information about positive or negative results was taken into consideration. However, a multicenter phase III clinical trial comparing Scintimun ${ }^{\circledR}$ and ${ }^{99 m}$ Tc-HMPAO in diagnosing peripheral bone infections provided evidence of good agreement between the 2 methods and of the efficacious diagnostic ability of both tracers to differentiate infection from sterile inflammation. ${ }^{20}$

\section{Conclusions}

Single-photon emission computed tomography with conventional CT with radioisotope-labeled leukocytes is a useful, efficient, single-step test for LDIE diagnosis with high sensitivity and specificity, and a satisfactory overall predictive value of over $77 \%$.

\section{References}

1. Małecka B, Kutarski A. Lead-dependent infective endocarditis: An old problem, a new name. Cardiol J. 2010;17(2):205-210.

2. Deckx S, Marynissen T, Rega F, et al. Predictors of 30-day and 1-year mortality after transvenous lead extraction: A single-centre experience. Europace. 2014;16(8):1218-1225.

3. Habib G, Hoen B, Tornos P, et al; ESC Committee for Practice Guidelines. Guidelines on the prevention, diagnosis, and treatment of infective endocarditis (new version 2009): The Task Force on the Prevention, Diagnosis and Treatment of Infective Endocarditis of the European Society of Cardiology (ESC). Eur Heart J. 2009;30(19):2369-2413.

4. Habib G, Lancellotti P, Antunes MJ, et al; ESC Scientific Document Group. 2015 ESC Guidelines for the management of infective endocarditis. Eur Heart J. 2015;36(44):3075-3128.

5. Erba PA, Sollini M, Conti U, et al. Radiolabeled WBC scintigraphy in the diagnostic workup of patients with suspected device-related infections. JACC Cardiovasc Imaging. 2013;6(10):1075-1086.

6. Palestro CJ, Brown ML, Forstrom LA, et al. Society of Nuclear Medicine Procedure Guideline for ${ }^{99 m}$ Tc-exametazime (HMPAO)-labeled leukocyte scintigraphy for suspected infection/inflammation. Version 3.0, approved June 2, 2004. http://snmmi.files.cms-plus.com/ docs/Leukocyte_v3.pdf. Accessed October 2, 2018. 
7. Sarrazin JF, Philippon F, Trottier M, Tessier M. Role of radionuclide imaging for diagnosis of device and prosthetic valve infections. World J Cardiol. 2016;8(9):534-546.

8. Attia J. Moving beyond sensitivity and specificity: Using likelihood ratios to help interpret diagnostic tests. Australian Prescriber. 2003;26(5):111-113.

9. Jaeschke R, Cook D, Guyatt G. Ocena artykułów na temat testów diagnostycznych. Cz. II - metody określenia przydatności testu. Med Prakt. 1998;11:184-191.

10. Landis JR, Koch GG. The measurement of observer agreement for categorical data. Biometrics. 1977;33(1):159-174.

11. Małecka B, Ząbek A, Mertyna P, Boczar K, Rydlewska A, Lelakowski J. Infection of permanent pacing system with negative inflammatory markers. Pol Arch Med Wewn. 2014;124(5):272-273.

12. Downey BC, Juselius WE, Pandian NG, Estes NA, Link MS. Incidence and significance of pacemaker and implantable cardioverter-defibrillator lead masses discovered during transesophageal echocardiography. Pacing Clin Electrophysiol. 2011;34(6):679-683.

13. Lo R, D'Anca M, Cohen T, Kerwin T. Incidence and prognosis of pacemaker lead associated masses: A study of 1,569 transesophageal echocardiograms. J Invasive Cardiol. 2006;18(12):599-601.

14. Polewczyk A, Janion M, Podlaski R, Kutarski A. Clinical manifestations of lead-dependent infective endocarditis: Analysis of 414 cases. Eur J Clin Microbiol Infect Dis. 2014;33(9):1601-1608.
15. Polewczyk A, Jacheć W, Janion M, Podlaski R, Kutarski A. Lead-dependent infective endocarditis: The role of factors predisposing to its development in an analysis of 414 clinical cases. Pacing Clin Electrophysiol. 2015;38(7):846-856.

16. Polewczyk A, Jacheć W, Tomaszewski A, et al. Lead-related infective endocarditis: Factors influencing the formation of large vegetations. Europace. 2017;19(6):1022-1030.

17. Cautela J, Alessandrini S, Cammilleri S, et al. Diagnostic yield of FDG positron-emission tomography/computed tomography in patients with CEID infection: A pilot study. Europace. 2013;15(2):252-257.

18. Graziosi M, Nanni C, Lorenzini M, et al. Role of ${ }^{18} \mathrm{~F}-\mathrm{FDG}$ PET/CT in diagnosis of infective endocarditis in patients with an implanted cardiac device: A prospective study. Eur J Nucl Med Mol Imaging. 2014;41(8):1617-1623.

19. Juneau D, Golfam M, Hazra S, et al. Positron emission tomography and single-photon emission computed tomography imaging in the diagnosis of cardiac implantable electronic device infection: A systematic review and meta-analysis. Circ Cardiovasc Imaging. 2017;10(4). pii: e005772. doi:10.1161/CIRCIMAGING.116.005772.

20. Richter WS, Ivancevic V, Meller J, et al. ${ }^{99 m}$ Tc-besilesomab (Scintimun) in peripheral osteomyelitis: Comparison with ${ }^{99 m T c-l a b e l e d}$ white blood cells. Eur J Nucl Med Mol Imaging. 2011;38(5):899-910. 